ARTICLE

\title{
FLUKA Realistic Modeling of Radiation Induced Damage
}

\author{
Alberto FASSO ${ }^{1}$, Alfredo FERRARI ${ }^{2}$, George SMIRNOV ${ }^{2}$, Florian SOMMERER ${ }^{3}$ and Vasilis VLACHOUDIS ${ }^{2, *}$ \\ ${ }^{1} 13$ Passage Hamo, CH-1262 Eysins, Switzerland \\ ${ }^{2}$ EN Dept., CERN, CH-1211, Geneva-23, Switzerland \\ ${ }^{3}$ University Clinic and Heidelberg Ion Therapy Center Im Neuenheimer Feld 450, 69120 Heidelberg, Germany
}

\begin{abstract}
The prediction of the structural damage to materials under irradiation is essential to evaluate consequences due to long term employment of construction materials in nuclear reactors and charged particle accelerators. The present paper describes the implementation of radiation damage effects in the particle transport code FLUKA for all particles and compares the results with the numerical evaluations available in the literature. In contrast with the implementation in other codes, we decided not to employ the Lindhard partition function but rather rework the formulas for an equivalent partition function restricted in energy above a user defined damage threshold. The implementation is based on the integration of the "universal" potential using realistic form factors, allowing for calculation of the restricted nuclear energy losses $d E_{n u c}^{E \text { th }} / d x=N \int_{E t h}^{E \max } T \frac{d \sigma}{d T} d T$ for the recoils above a certain fraction of energy. The integration was performed in the "reduced energy" frame to remove any dependence on the projectile and target nucleus. The results obtained were used in a two-dimensional fit with a tolerance less than $5 \%$ spanning over nine orders of magnitude. Based on the fitting, during run-time FLUKA is able to predict in an accurate way the number of recoils (and subsequent sub-cascade of the recoils) which has the power to induce damage to the material. Finally the conversion to $d p a$ is performed by using a modified Kinchin-Pease damage model and taking into account the effect on the displacement efficiency for higher recoil energies due to recombination and migration of the Frenkel pairs. All charged particles including leptons, hadrons and heavy ions, benefit from the algorithm. However for neutron interactions, photo-nuclear interactions, Bremsstrahlung, pair production, Compton scattering the recoil nucleus is either calculated analytically or sampled from a distribution and is further subject to the above algorithm for the damage estimation.
\end{abstract}

KEYWORDS: FLUKA, DPA, NIEL, radiation damage

\section{Introduction}

FLUKA $^{1,2)}$ is a general purpose Monte Carlo code capable of handling all radiation components from thermal energies (for neutrons), or $1 \mathrm{keV}$ (for all other particles) till cosmic ray energies and can be applied in many different fields. The validity of the physical models implemented in FLUKA has been benchmarked against a variety of experimental data over a wide energy range, from accelerator data to cosmic ray showers in the Earth atmosphere. FLUKA is widely used for studies related both to basic research and to applications in particle accelerators, radiation protection and dosimetry, including the specific issue of radiation damage in space missions, radiobiology (including radiotherapy) and cosmic ray calculations.

The effects of radiation on the properties of solids are of significant interest in scientific and technological contexts. Moreover, as the power of particle accelerators is increasing $^{3)}$ the prediction of the structural damage to materials under irradiation is essential to evaluate consequences due to long term employment. Since FLUKA is used for the protection studies of the LHC collider ${ }^{4)}$ it was recently enhanced

*Corresponding author, E-mail: Vasilis.Vlachoudis@cern.ch

(C) 2011 Atomic Energy Society of Japan, All Rights Reserved. with the capability of making realistic predictions of radiation induced damage to materials. In the following sections this implementation will be described and compared with the predictions of other codes.

\section{Displacement Damage}

The unit that is frequently used for measuring the radiation damage is the Displacement Per Atom (dpa). It is a measure of the amount of radiation damage in irradiated materials. For example, $3 d p a$ means each atom in the material has been displaced from its site within the structural lattice of the material an average of 3 times. Displacement damage can be induced by all particles produced in the hadronic cascade, including high energy photons. The latter, however, have to initiate a reaction producing charged particles, neutrons or ions. The dpa quantity is directly related with the total number of defects or Frenkel pairs $N_{F}$

$$
d p a=\frac{A}{N_{A} \rho} N_{F}
$$

where $\rho$ is the density in units of $\mathrm{g} / \mathrm{cm}^{3}, A$ is the mass number and ${ }_{\mathrm{NA}}$ is the Avogadro number. 


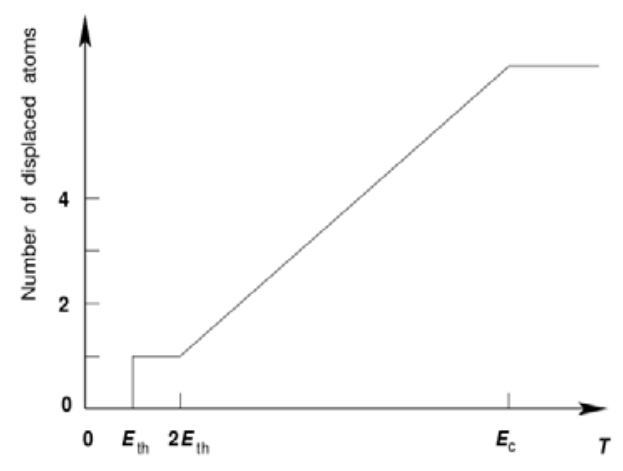

Fig. 1 Schematic relation between the number of displaced atoms in the cascade and the kinetic energy $T$ of the PKA

\section{Frenkel Pairs}

A Frenkel pair $N_{F}$ (defect or disorder), is a compound crystallographic defect in which an interstitial lies near the vacancy. A Frenkel defect forms when an atom or ion leaves its place in the lattice creating a vacancy, and lodges nearby becoming an interstitial. The first practical formula for the calculations of $N_{F}$ was suggested by Kinchin-Pease model $(\mathrm{K} \& \mathrm{P}){ }^{6}{ }^{6}$ It was later modified by Norgert, Robinson and Torrens (NRT), ${ }^{7)}$ with the total number of defects $N_{N R T}$ being:

$$
N_{N R T} \equiv N_{F}=\kappa \frac{\xi(T) T}{2 E_{t h}}
$$

where $N_{N R T}$ is the number of defects by Norgert, Robinson and Torrens, $\kappa=0.8$ is the displacement efficiency, $T$ is the kinetic energy of the primary knock-on atom (PKA), $\xi(T)$ is the Lindhard partition function according to the LSS theory, ${ }^{8)} E_{t h}$ is the displacement damage threshold. The assumptions used for deriving this expression are: (i) the cascade is created by a sequence of two-body elastic collisions between atoms; (ii) in the collision process, the energy transferred to the lattice is zero; (iii) for energies below the cut-off energy $E_{c}$ the electronic stopping is ignored and only atomic collisions take place; (iv) above the cut-off energy $E_{c}$, no additional displacement occurs, $(v)$ the energy transfer cross section is given by the hard-sphere model. Therefore the number of defects $v(T)$ are:

$$
\begin{array}{ll}
v(T)=0 & \text { for } 0<T \leq E_{t h} \text { (phonons), } \\
v(T)=1 & \text { for } E_{t h}<T \leq 2 E_{t h}, \\
v(T)=T / 2 E_{t h} & \text { for } 2 E_{t h}<T \leq E_{c}, \\
v(T)=E_{c} / 2 E_{t h} & \text { for } T>E_{c .} .
\end{array}
$$

Figure 1 shows the schematic relation between the number of displaced atoms in the cascade and the kinetic energy $T$ of the PKA.

\section{Description of Main Ingredients}

The factor $2 E_{t h}$ is due to the hard sphere model, which assumes that the energy is equally shared between the two atoms on each collision.

The threshold displacement energy $E_{t h}$ is the average minimum energy over all crystallographic directions to pro-
Table 1 Typical values used by NJOY

\begin{tabular}{cccc}
\hline Material & $E_{\text {th }}(\mathrm{eV})$ & Material & $E_{\text {th }}(\mathrm{eV})$ \\
\hline $\mathrm{Li}$ & 10 & $\mathrm{Co}$ & 40 \\
$\mathrm{C}$ in SiC & 20 & $\mathrm{Ni}$ & 40 \\
Graphite & $30-35$ & $\mathrm{Cu}$ & 40 \\
$\mathrm{Al}$ & 27 & $\mathrm{Nb}$ & 40 \\
$\mathrm{Si}$ & 25 & $\mathrm{Mo}$ & 60 \\
$\mathrm{Mn}$ & 40 & $\mathrm{~W}$ & 90 \\
$\mathrm{Fe}$ & 40 & $\mathrm{~Pb}$ & 25 \\
\hline
\end{tabular}

duce a defect. Its value is of the order of tens of eV depending on the material. Table 1 shows some typical values as used by the NJOY code. ${ }^{9}$

The displacement efficiency $\kappa=0.8$ deviates from the hard sphere model (K\&P), ${ }^{6}$ and compensates for the forward scattering in the displacement cascade. The displacement efficiency $\kappa$ can be considered as independent on $T$ only in the range of $T \leq 1-2 \mathrm{keV}$. At higher energies, the development of collision cascades results in defect migration and recombination of Frenkel pairs due to overlapping of different branches of a cascade which translates into decay of $\kappa(T)$. From molecular dynamics (MD) simulations ${ }^{10,11)}$ of the primary cascade the number of surviving displacements, $N_{M D}$, normalized to the number of those from NRT model, $N_{N R T}$, decreases down to the values $0.2-0.3$ at $T \approx 20-100 \mathrm{keV}$. The efficiency in question only slightly depends on atomic number $Z$ and the temperature. Therefore, in our model we employed an approximation of the results of MD in the following form Ref. 5):

$$
N_{M D} / N_{N R T}=0.3-1.3\left(-\frac{9.57}{X}+\frac{17.1}{X^{4 / 3}}-\frac{8.81}{X^{5 / 3}}\right)
$$

where $X \equiv 20 T$ in $\mathrm{keV}$.

\section{Lindhard Partition Function $\xi(T)$}

In the NRT model the product $\xi(T) T$ is closely related to the frequently used magnitude NIEL which is an acronym for non-ionizing energy loss. In the general case, NIEL is determined by the spectrum of recoil particles (atoms) $d \sigma / d T$ integrated within the interval of recoil energies from 0 to the maximum recoil energy $T_{\max }=\gamma E$, with $\gamma=4 m M /(m+M)^{2}$.

$$
N I E L(E)=\frac{N_{A}}{A} \int_{0}^{{ }^{E}} \xi(T) T\left(\frac{d \sigma}{d T}\right)_{E} d T
$$

where $E$ is the energy of the projectile, $m$ and $M$ are the masses of the projectile and material atom, respectively. The value of $\xi(T)$ gives the fraction of the stopping power $S(T)$ that goes into NIEL. Therefore $\xi(T)=S_{n}(T) / S(T)$, with the $S_{n}(T)$ being the nuclear stopping power.

Based on the assumptions that (i) electrons do not produce recoil atoms; (ii) the atomic binding term is negligible; (iii) the energy transfer to electrons is small in a relative measure; (iv) the $T$ of the PKA is small compared with the projectile kinetic energy $E$, Lindhard ${ }^{8,12)}$ approximated the value of $\xi(T)$ as 


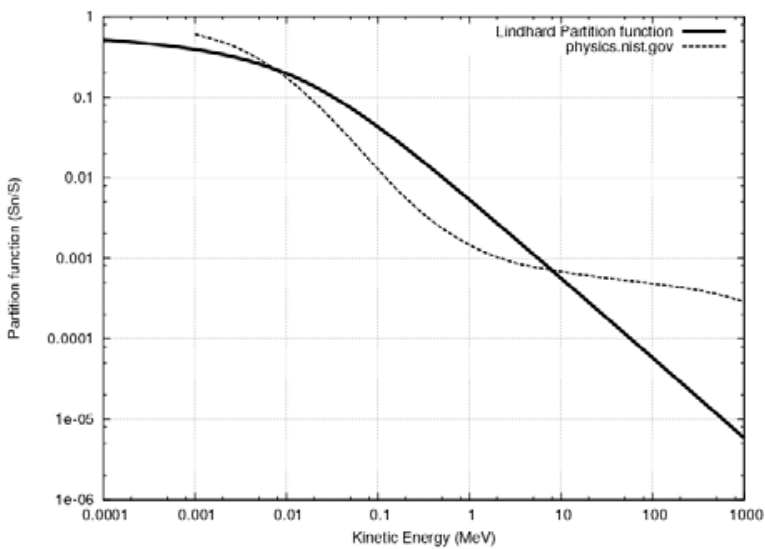

Fig. 2 Comparison of partition function for alpha particles impinging on Silicon as a function of energy. Lindhard approximation (solid line) and astar program ${ }^{13)}$ from the National Institute of Standards and Technology partition function (dashed line)

$$
\begin{aligned}
& \xi(T)=\frac{1}{1+F_{L} \cdot\left(3.4008 \cdot \varepsilon(T)^{1 / 6}+0.40244 \cdot \varepsilon(T)^{3 / 4}+\varepsilon(T)\right)} \\
& F_{L}=30.724 \cdot Z_{1} \cdot Z_{2} \sqrt{Z_{1}^{2 / 3}+Z_{2}^{2 / 3}} \\
& \varepsilon(T)=\frac{T}{0.0793 \frac{Z_{1}^{2 / 3} \cdot \sqrt{Z_{2}}}{\left(Z_{1}^{2 / 3}+Z_{2}^{2 / 3}\right)^{3 / 4}} \cdot \frac{\left(A_{1}+A_{2}\right)^{3 / 2}}{A_{1}^{3 / 2} \sqrt{A_{2}}}}
\end{aligned}
$$

where subscript 1 refers to the projectile, 2 to the target, $Z$ is the charge, $A$ is the mass number. A nice feature of this approximation is that it can handle any projectile $Z_{1}, A_{1}$ and in general an arbitrary charged particle. However, when the results of Eq. (5) are compared with the more recent approximations of the nuclear $S_{n}$ and electronic $S_{e}$ stopping power, ${ }^{13)}$ Eq. (5) reproduces quite well the partition function $S_{n} /\left(S_{e}+S_{n}\right)$ for low energies $(T<1 \mathrm{keV})$ but it suffers serious discrepancies for higher energies (Fig. 2). These discrepancies are due to the assumptions (see above) made by Lindhard in deriving the formula.

\section{Nuclear Stopping Power}

For the estimation of $\underline{d p a}$ with the NRT model, Eq. (2) and Eq. (4) make use of the NIEL and the Lindhard partition function $\xi(T)$. Both are based on the unrestricted in energy nuclear stopping power

$$
S_{n}(E)=N \int_{0}^{\gamma E} T\left(\frac{d \sigma}{d T}\right)_{E} d T
$$

where $N$ is the atomic density, $T$ is the energy transfer during ion-solid interaction, $d \sigma / d T$ is the differential scattering cross-section.

\section{Restricted Nuclear Stopping Power}

With the above approach we are overestimating the $d p a$ since we are summing up also all recoils with $T<E_{\text {th }}$ that are smaller than the damage threshold $E_{t h}$. A more appropriate estimation of the $d p a$ will imply the use of the restricted in energy nuclear stopping power for recoils with energy $T$ above the damage threshold $E_{t h}$.

$$
S_{n}\left(E, E_{t h}\right)=N \int_{E t h}^{\gamma E} T\left(\frac{d \sigma}{d T}\right)_{E} d T
$$

which is equivalent to

$$
\frac{1}{\rho} S_{n}\left(E, E_{t h}\right)=-2 \pi N \int_{0}^{b \max } b \frac{d b}{d \theta} W(\theta, E) d b
$$

where $\theta$ is the deflection angle, $W(\theta, E)=\gamma E \sin ^{2}(\theta / 2)$ is the energy transfer to the recoil atom, $b$ is the impact parameter, $b_{\max }$ is the maximum impact parameter corresponding to energy transfer $E_{t h}=W_{\min }\left(\theta_{\min }, E\right)$.

Therefore, $\theta_{\min }=2 \arcsin \left(\sqrt{\frac{E_{t h}}{\gamma E}}\right)$.

The scattering deflection angle $\theta$ can be calculated by

$$
\theta=\pi-2 \int_{\mathrm{r}_{\min }}^{\infty} \frac{p d r}{r^{2} \sqrt{1-\frac{V(r)}{E_{c m s}}-\frac{p^{2}}{r^{2}}}}
$$

where $V(r)$ is the interatomic potential of the two atoms, $E_{c m s}$ is the energy in the CMS, $r_{\min }$ is the minimum impact parameter, and $p$ is the impact parameter.

To find the maximum impact parameter $r_{\max }$ corresponding to a minimum recoil of $E_{t h}$ we have to numerically solve Eq. (9) for $\theta=\theta_{\text {min. }}$ Either it can be done iteratively by numerical evaluation of the integral Eq. (9) or by using the magic scattering formula from Biersack-Haggmark ${ }^{14)}$ which provides a fitting to the $\sin ^{2}(\theta / 2)$. It is obvious that solving for $\theta_{\min }$ and subsequently calculating the restricted nuclear losses $S_{n}\left(E, E_{t h}\right)$ poses a serious penalty in CPU-time, therefore, an approximate solution has to be found.

\section{Approximation to Restricted Nuclear Energy Losses}

Our approximation of the restricted nuclear energy losses is based on the approximation of Ziegler ${ }^{15)}$ of the unrestricted reduced nuclear stopping power in $\left(\mathrm{MeV} / \mathrm{g} / \mathrm{cm}^{2}\right)$

$$
\frac{1}{\rho} S_{n}(T)=\frac{5105.3 Z_{1} Z_{2} \hat{S}_{n}(\varepsilon)}{\left(Z_{1}^{0.23}+Z_{2}^{0.23}\right)\left(1+\frac{M_{2}}{M_{1}}\right) A}
$$

using the reduced stopping power and energy

$$
\begin{aligned}
& \text { if } \varepsilon<30 \quad \hat{S}_{n}(\varepsilon)=\frac{0.5 \ln (1+1.1383 \varepsilon)}{\varepsilon+0.01321 \varepsilon^{0.21226}+0.19593 \sqrt{\varepsilon}}, \\
& \text { if } \varepsilon \geq 30 \quad \hat{S}_{n}(\varepsilon)=\frac{\ln (\varepsilon)}{2 \varepsilon} .
\end{aligned}
$$

The reduced energy $\varepsilon$ is given by ( $T$ in $\mathrm{keV}$ )

$$
\varepsilon=\frac{32.536 T}{\left(Z_{1}^{0.23}+Z_{2}^{0.23}\right)\left(1+\frac{M_{1}}{M_{2}}\right) Z_{1} Z_{2}} .
$$

The important features of this approximation are that it 


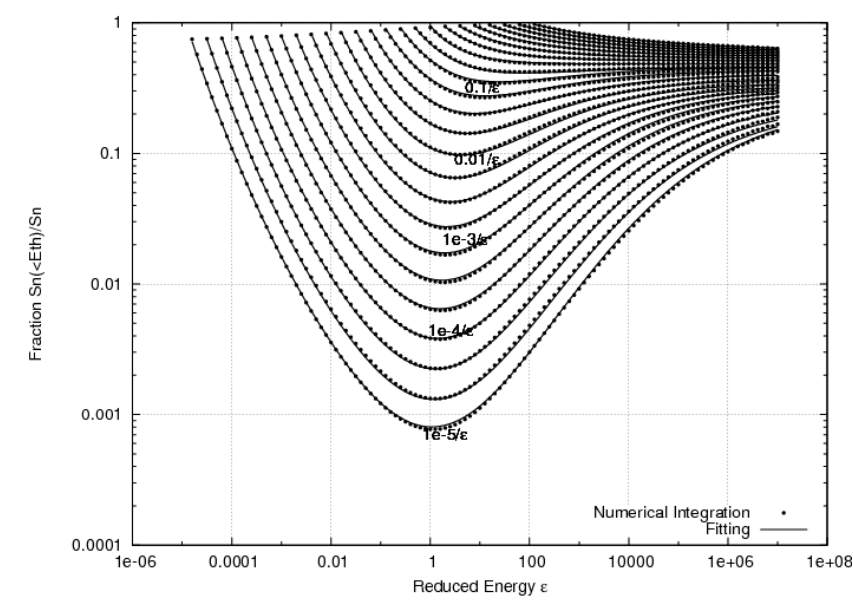

Fig. 3 Comparison of the fraction $\xi_{r}\left(E, E_{t h}\right)$ restricted partition function from the fitting routine (solid) and as numerically approximated (points) versus the reduced energy $\varepsilon$ and for various $\sin ^{2}\left(\theta_{\min } / 2\right)$

can be employed for any projectile-target combination as well with any charged particle. Moreover, is quite accurate $\sim 1 \%$ for $\varepsilon<1$ and to within 5\% or better for $\varepsilon>3$.

For the fitting we had to solve numerically the restricted reduced $^{\mathrm{a}}$ nuclear stopping power over a wide range of $\sin ^{2}\left(\theta_{\min } / 2\right)$ and reduced energy $\varepsilon$. Subsequently we fitted the fraction of the restricted reduced nuclear stopping power $\xi_{r}\left(E, E_{t h}\right)$ that falls below the threshold $E_{t h}=W\left(\theta_{\min }, E\right)$, over the total reduced stopping power as a function of the $\sin ^{2}\left(\theta_{\min } / 2\right)$ and reduced energy $\varepsilon$. The fitting formula we used is:

$$
\begin{aligned}
& X=\log _{10}\left[\sin ^{2}\left(\theta_{\min } / 2\right) \varepsilon\right] \\
& Y=\log _{10}[\varepsilon]
\end{aligned}
$$

for $X<0.3$ we use a 5th order polynomial fit to $\xi_{r}\left(E, E_{t h}\right)$ with coefficients $A, B, C, D, E, F$ as a function of $Y$ and the fraction of the restricted reduced nuclear losses over the unrestricted is given by

$$
\xi_{r}\left(E, E_{t h}\right)=10^{\exp (-\exp (-A X+B)+C)+\exp (-D X+E)-F}
$$

for $X \geq 0.3$ we use a 3rd order polynomial fit to $\xi_{r}\left(E, E_{t h}\right)$, and the fraction is given by

$$
\xi_{r}\left(E, E_{t h}\right)=10^{\exp (-A X+B)-C} .
$$

The fitting extends over nine orders of magnitude in reduced energy $\varepsilon$, and seven orders of magnitude in $\sin ^{2}\left(\theta_{\min } / 2\right)$. It has very good accuracy with a maximum error $<5 \%$ close to $X=0.3$. Figure 3 shows the comparison of the $\xi_{r}\left(E, E_{t h}\right) r e-$ stricted partition function from the fitting routine and as it is numerically approximated.

\section{FLUKA Implementation}

In FLUKA we decided to have uniform implementation for all particles and with minimum input from the user. Therefore, the only parameter the user is requested to supply

\footnotetext{
${ }^{a}$ restricted for $E>E_{t h}$, and reduced working the reduced energy
} frame as given by Eq. (12).

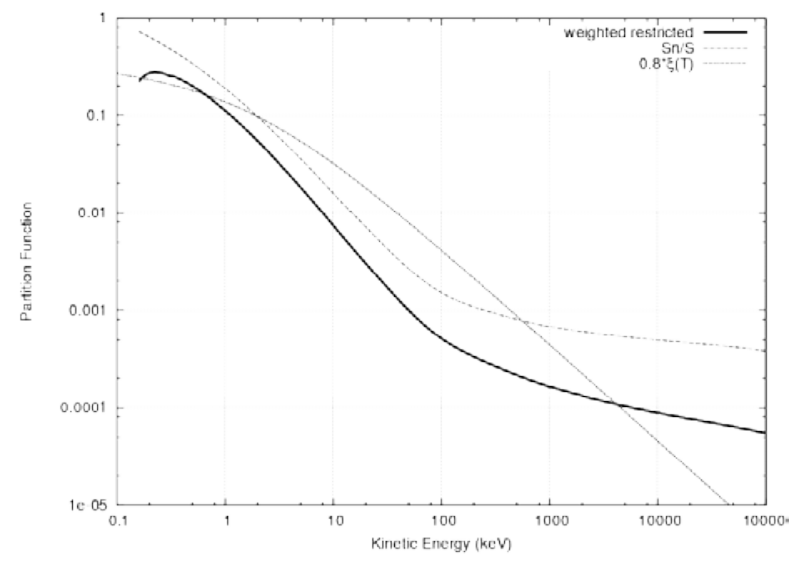

Fig. 4 Comparison of the weighted restricted fraction as given from Eq. (18) compared with the unrestricted $S_{n} / S$ and the Linhard partition function $\xi(T)$ multiplied with the displacement efficiency $\kappa=0.8$ from the NRT model

is the damage threshold $E_{t h}$ for all materials in the simulation. To this end, we reworked all formulas to calculate the restricted in energy nuclear losses, which above the damage threshold are only responsible for dpa. A two-dimensional integral is solved numerically and fitted with a two-dimensional function having a tolerance less than 5\% spanning over nine orders of magnitude. Based on the fitting, for any impinging particle, during run-time FLUKA appropriately evaluates the number of recoils (and subsequent sub-cascade of the recoils) which have the power to induce damage to the material. In other words, a recoil nucleus is treated as a new projectile. As it will be described below the treatment is valid from $1 \mathrm{keV}$ for all particles (thermal for neutrons) up to cosmic ray energies of $\mathrm{PeV}$.

\section{Charged Particles}

During the transport of all charged particles and heavy ions the dpa estimation is based on the restricted nuclear stopping power while for NIEL on the unrestricted one. Therefore, for every particle above the transport threshold and for every Monte Carlo step, the number of defects is calculated based on a modified Eq. (4) as the multiple integral

$$
N(E)=\int_{E_{t h}}\left[\xi_{r}\left[T, E_{t h}\right)\left(\frac{d \sigma}{d T}\right)_{E_{E_{t h}}^{\gamma}}^{\gamma / T} \kappa\left(T^{\prime}\right) \xi_{r}\left(T^{\prime}, E_{t h}\right) T^{\prime}\left(\frac{d \sigma}{d T^{\prime}}\right)_{T} d T^{\prime}\right] d T
$$

where the prime symbol refers to the target material which in the sub-cascade becomes the new projectile. With Eq. (18), we are using the more accurate estimation of the partition function for the initial particle and we include the first sub cascade branch of the recoil particles. The calculation is based on the restricted partition function weighted also with the modified displacement efficiency $\kappa(T)$ that takes into account the branch overlaps and recombination. Higher order sub cascades are rapidly reduced in energy and fall into the validity of the Lindhard assumptions, therefore we can safely assume that the nuclear stopping power is the dominant component. For the particles falling below the transport threshold of FLUKA (1 keV) we use a modified Eq. (3) for 


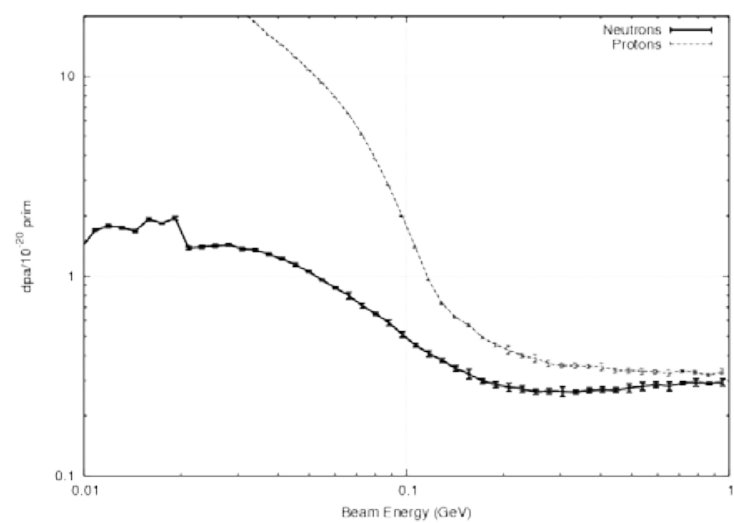

Fig. 5 Maximum peak of $d p a$ from a pencil neutron and proton beam versus beam energy on a diamond target. Is visible the discontinuity at $20 \mathrm{MeV}$ for neutrons due to the different special group treatment applied at low energies.

the $d p a$ estimation. Figure 4 shows the FLUKA prediction as weighted restricted partition function from Eq. (18) for protons on diamond, compared with the unrestricted nuclear losses and the Lindhard partition function $\xi(T)$ multiplied with the displacement efficiency $\kappa=0.8$ from the NRT model. At low energies $(<1 \mathrm{keV})$ the Linhdard partition function and the NIEL approximation have small differences, however for higher energies the weighted restricted nuclear stopping power is significantly lower than the NIEL approximation.

After any interaction all recoil particles if they are above the transport threshold set by the user, they are treated as new projectiles and they are subject to the above treatment. For all particles including the recoils falling below the transport threshold the NRT model Eq. (2) is used, which for low thresholds $\sim 1 \mathrm{keV}$ as we discussed before it reproduces quite well the unrestricted NIEL.

\section{Neutrons}

FLUKA uses the multi-group approach for the low-energy neutrons $(<20 \mathrm{MeV})$. The neutron cross sections libraries are pre-processed with the $\mathrm{NJOY}^{9)}$ program which provides group-to-group (down/up) scattering probabilities. Since the neutron interactions with the group approach are not treated explicitly there is no possibility of calculating the recoil energy, apart from few exceptions were point-wise treatment is used. Therefore during the processing of the libraries we include the NIEL information as it is provided by NJOY and it is used as such for the $d p a$, generating an overestimation of the dpa. The effect is visible as a small discontinuity (Fig. 5) exactly at the matching energy of $20 \mathrm{MeV}$ where the models stop and the group approach is used. In the future, when the low energy neutron point-wise treatment will be implemented for all materials in FLUKA this artifact will disappear.

\section{Pair Production and Bremsstrahlung}

There are two special cases of interactions, the pair $\mathrm{e}^{+}, \mathrm{e}^{-}$ production by photons and the Bremsstrahlung ${ }^{5,16,17)}$ both normally have a small momentum transfer to the target which has the capability to generate $d p a$. However the exact calculations of the nuclear recoil spectra are extremely involved and hardly can be used in practical simulations. On the other hand there are number of derivations of the cross section $d \sigma / d p$ of the recoil momentum $p$.

For the Bremsstrahlung the cross section can be written $\mathrm{as}^{18)}$

$$
\frac{d \sigma}{d p_{\perp}}=\frac{32 a(Z a)^{2}}{k p_{\perp}^{3}}\left[1-\frac{k}{E}+\frac{1}{2}\left(\frac{k}{E}\right)^{2}\right] \ln \left(\frac{p_{\perp}}{m_{e}}\right)
$$

where $k$ is the energy of the Bremsstrahlung photon, $m_{e}$ is the electron mass, $E$ is the incident electron energy.

For the pair production an approximation of the cross section $^{16)}$ is given by

$$
\frac{d \sigma}{d p}=\frac{0.183 \cdot 10^{-2} Z^{2}}{p^{3}}(\ln (p)+0.5)
$$

Both Eq. (19) and Eq. (20) can be written in the same approximate way as

$$
\frac{d \sigma}{d p} \propto \frac{\ln (p / c)}{p^{3}}
$$

where $c$ is a constant which corresponds to the minimum recoil momentum. Therefore, in our implementation, when a pair production or Bremsstrahlung event occurs we sample the recoil momentum from the Eq. (21) using as lower and upper momentum limits those dictated by the interaction kinematics. Even though the Eq. (21) can be integrated analytically the inverse of the integral cannot be solved analytically, therefore direct sampling is not possible, we are using the rejection technique from a function that resembles Eq. (21) with an efficiency of $80 \%$. For high photon energies, the recoil of the pair production has very low limits much lower than the damage threshold $E_{t h}$. Therefore, when $d p a$ scoring is requested, a biased sampling is performed only for momentum $p$ which corresponds to recoils energy higher than $E_{t h}$.

\section{Performances}

Equation (18) is quite complicated and it has a serious penalty in CPU time, therefore it is tabulated at initialization time for all predefined particles of FLUKA as well as for the primary heavy ion if any. Due to memory constraints we cannot tabulate Eq. (18) for all possible recoil atoms, therefore for the other ions the integral is calculated numerically during transport time, however with lower precision.

When a particle falls below the transport threshold set in FLUKA by the user, the NRT formula is used as an approximation. Therefore it is strongly recommended that users perform a calculation with transport limits as low as possible $(\sim 1 \mathrm{keV})$ in order to benefit from the more accurate estimation of our implementation. Table 2 shows the comparison of the calculation ${ }^{19)}$ two cases (i) $1 \mathrm{GeV}$ protons on $3 \mathrm{~mm}$ thick Fe target with a beam area of $1 \mathrm{~cm}^{2}$, and (ii) $320 \mathrm{MeV} / \mathrm{A}^{238} \mathrm{U}$ onto $1 \mathrm{~mm}$ Be target with a beam area of $9 \mathrm{~cm}^{2}$. The results from SRIM, ${ }^{20)}$ PHITS, ${ }^{21)} \mathrm{MCNPX}^{22)}$ are 
Table 2 dpa calculation comparison for $10^{20}$ beam particles

\begin{tabular}{lccccc}
\hline Case & SRIM & PHITS & MCNPX & MARS & FLUKA \\
\hline p on Fe & 0.0118 & 0.296 & 0.335 & 0.871 & 0.28 \\
${ }^{238} \mathrm{U}$ on Be & 2.97 & 0.0502 & - & 2.13 & 1.68 \\
\hline
\end{tabular}

courtesy of Susana Reyes those from MARS15 ${ }^{23)}$ are a courtesy of N. Mokhov. ${ }^{19)}$

Figure 6 shows a comparison of our model with the prediction of other simulation codes, for a ${ }^{76} \mathrm{Ge}$ ion beam of $130 \mathrm{MeV} / \mathrm{A}$ uniformly impacting on a $\mathrm{R}=0.03568 \mathrm{~cm}$ disc of $\mathrm{W}$ target with $1.2 \mathrm{~mm}$ thickness. Our model using the restricted energy losses (Eqs. (16), (17)) together with the modified displacement efficiency (Eq. (3)) and the double integration (Eq. (18)) shows lower dpa values compared with the TRIM_2 and MARS, but higher than those from PHITS courtesy of Yosuke Iwamoto. The difference on the depth of the Bragg peak is due to the introduction in FLUKA of the Barkas and Bloch corrections for the ionization losses.

\section{Summary}

We described our formalism for calculating damage to materials and the implementation into FLUKA of the NIEL and $d p a$ evaluation. The user has to define the only free parameter that is requested, which is the damage threshold $\mathrm{E}_{\mathrm{th}}$, for each material under investigation. FLUKA is able to predict in an accurate way the number of recoils which have the power to induce damage to the material. Finally the conversion to $d p a$ is performed by using a modified Kinchin-Pease damage model and taking into account the effect on the displacement efficiency for higher recoil energies due to recombination and migration of the Frenkel pairs. In our formalism we treat all particles with the same model with the exceptions, of low-energy $(<20 \mathrm{MeV})$ neutron interactions, Bremsstrahlung and pair production. We are confident that our model is self consistent and behaves the same way for all particle energies. Therefore a material tested for damage at low energies (MeV) and simulated with FLUKA may be justly compared with the prediction for the same material under higher energies (TeV) of the LHC.

\section{Acknowledgments}

The authors would like to thank Ralf Assmann for the interest in the project and numerous discussions, Alexander Ryazanov for the valuable discussions concerning the damage effects, Steffen Mueller for testing and validating the code, Francesco Cerutti for proof reading the paper.

\section{References}

1) A. Fassò, A. Ferrari, J. Ranft, P. R. Sala, FLUKA: a multi-particle transport code, CERN-2005-10 (2005), INFN/TC_05/11, SLAC-R-773.

2) G. Battistoni, S. Muraro, P. R. Sala, F. Cerutti, A. Ferrari, S. Roesler, A. Fassò, J. Ranft, "The FLUKA code: Description and benchmarking," Proceedings of the Hadronic Shower Simulation Workshop 2006, Fermilab 6-8 September 2006, M.

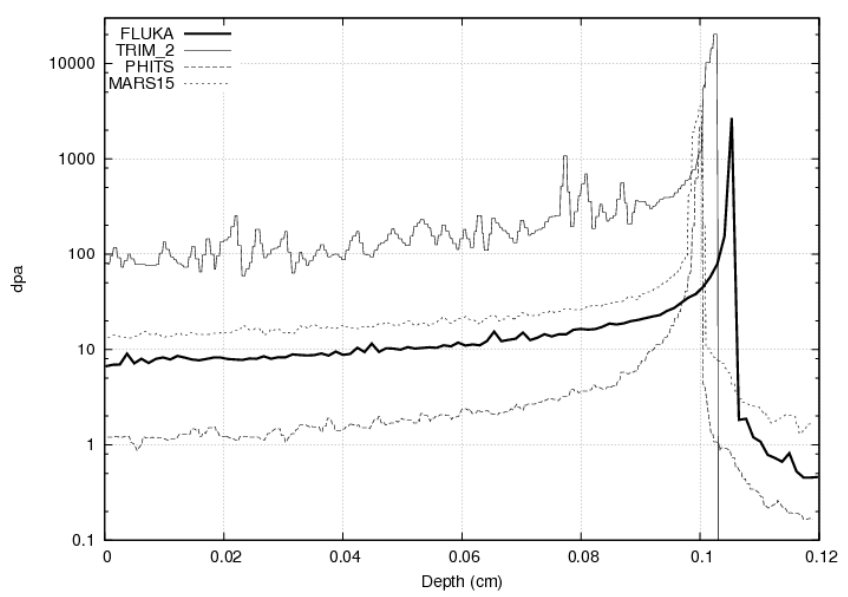

Fig. 6 dpa calculation of a ${ }^{76} \mathrm{Ge}$ ion pencil beam of $130 \mathrm{MeV} / \mathrm{A}$ uniform in $\mathrm{R}=0.03568 \mathrm{~cm}$ disc of $\mathrm{W}$ target with $1.2 \mathrm{~mm}$ thickness with various Monte Carlo codes

Albrow, R. Raja (Eds.), AIP Conference Proceeding 896, 31-49 (2007).

3) R. Assmann et al., "Requirements for the LHC Collimation System," Proc. 8th European Particle Accelerator Conference (EPAC02), La Vilette, Paris, France 3-7 June 2002 - E.P.S., Geneva, pp 197 (2002). LHC Project Report 599.

4) V. Vlachoudis et al., "Consequences of Regular and Irregular Beam Impact on the LHC Collimators," Monte Carlo 2005 Topical Meeting, Chattanooga, Tennessee, USA, April 17-21 (2005).

5) G. Smirnov et al., "Simulating radiation damage effects in LHC collimators," CERN, September 5, 2007, Internal Note (2008).

6) G. H. Kinchin, R. S. Pease, Rep. Progr. Phys., 18, 1 (1955).

7) M. J. Norgett, M. T. Robinson, I. M. Torrens, Nucl. Eng Des., 33, 50 (1975).

8) J. Lindhard, V. Nielsen, M. Scharff, P. V. Thomsen, Mat. Fys. Medd. Dan. Vid. Selsk., 33, No. 10 (1963).

9) R. E. MacFarlane, R. M. Boicourt, "NJOY: A neutron and photon cross-section processing system," Trans. Am. Nucl. Soc., 22, 720 (1975).

10) R. E. Stoller, J. Nucl. Mat., 276, 22 (2000).

11) D. J. Bacon, F. Gao, Y. N. Osetsky, J. Comp.-Aided Mat. Des., 6, 225 (1999)

12) M. T. Robinson, "Radiation Induced Voids in Metals," (Eds.) J. W. Corbett L. C. Ianiello, CONF-710601, 397-429 (1972).

13) M. J. Berger, J. S. Coursey, M. A. Zucker, J. Chang, "Stopping-Power and Range Tables for Electrons, Protons, and HeHelium Ions,” http://www.nist.gov/physlab/data/star/index.cfm

14) J. P. Biersack, "Computer Simulations of Sputtering," Nucl. Instr. Meth. Phys. Res., B27, 21-36 (1987).

15) International Commission of Radiation Units and Measurements (ICRU), Stopping Powers and Ranges for Protons and Alpha Particles, ICRU Report 49 (1993).

16) H. A. Bethe, Proc. Cambridge Phil. Soc., 30, 524 (1934).

17) R. Jost, J. M. Luttinger, M. Slotnik, Phys. Rev., 80, 189 (1950).

18) A. I. Akhiezer, V. B. Berestetskii, Quantum Electrodynamics, NAUKA, Moscow (1981).

19) N. Mokhov et al., "Simulation and verification of DPA in materials," Workshop of Applications of High-Intensity Proton Accelerators, Fermilab, Oct 19-21, 2009 (2009).

20) J. Ziegler, "SRIM-2003", Nucl. Instr. Meth. Phys. Res., B219-220, 1027-1036 (2004).

21) H. Iwase, K. Niita, T. Nakamura, "Development of Gener- 
al-Purpose Particle and Heavy Ion Transport Monte Carlo Code," J. Nucl. Sci. Technol. 39, 1142 (2002).

22) H. G. HUGHES et al., "MCNPX for Neutron-Proton Transport," Mathematical and Computation Topical Meeting,
American Nuclear Society, Madrid Spain, Sept 27-30 (1999).

23) N. V. Mokhov, S. I. Striganov, "MARS15 Overview," AIP Conf. Proc., 896, 50-60 (2007). 